\title{
Standing genetic variation and compensatory evolution in transgenic organisms: a growth-enhanced salmon simulation
}

\author{
Robert N. M. Ahrens • Robert H. Devlin
}

Received: 28 June 2010/Accepted: 9 September 2010/Published online: 29 September 2010

(C) The Author(s) 2010. This article is published with open access at Springerlink.com

\begin{abstract}
Genetically modified strains usually are generated within defined genetic backgrounds to minimize variation for the engineered characteristic in order to facilitate basic research investigations or for commercial application. However, interactions between transgenes and genetic background have been documented in both model and commercial agricultural species, indicating that allelic variation at transgene-modifying loci are not uncommon in genomes. Engineered organisms that have the potential to allow entry of transgenes into natural populations may cause changes to ecosystems via the interaction of their specific phenotypes with ecosystem components and services. A transgene introgressing through natural populations is likely to encounter a range of natural genetic variation (among individuals or sub-populations) that could result in changes in phenotype, concomitant with effects on fitness and ecosystem consequences that differ from that seen in the progenitor transgenic strain. In the present study, using a growth hormone transgenic salmon example, we have modeled selection of
\end{abstract}

R. N. M. Ahrens

Fisheries Centre, University of British Columbia, 2202 Main Mall, Vancouver, BC V6T 1Z4, Canada e-mail: ahrens@zoology.ubc.ca

R. H. Devlin ( $\square)$

Fisheries and Oceans Canada, 4160 Marine Drive, West Vancouver, BC V7V 1N6, Canada

e-mail: robert.devlin@dfo-mpo.gc.ca modifier loci (single and multiple) in the presence of a transgene and have found that accounting for genetic background can significantly affect the persistence of transgenes in populations, potentially reducing or reversing a "Trojan gene" effect. Influences from altered life history characteristics (e.g., developmental timing, age of maturation) and compensatory demographic/ecosystem controls (e.g., density dependence) also were found to have a strong influence on transgene effects. Further, with the presence of a transgene in a population, genetic backgrounds were found to shift in non-transgenic individuals as well, an effect expected to direct phenotypes away from naturally selected optima. The present model has revealed the importance of understanding effects of selection for background genetics on the evolution of phenotypes in populations harbouring transgenes.

Keywords Transgenic - Background genetics Compensatory evolution - Modifier - Fitness - Risk · Salmon · Growth $\cdot$ GH $\cdot$ Trojan gene

\section{Introduction}

Genetically engineered fish have now been in development for more than 25 years (Zhu et al. 1985) for use as basic research models and for commercial 
application. A major focus of research has been the production of transgenic fish that are growth enhanced compared to founder strains, which in some cases (e.g., mud loach and coho salmon) has resulted in very large increases in body size of up to 35- to 37-fold at specific ages (Devlin et al. 1994; Du et al. 1992; Martinez et al. 1996; Nam et al. 2001; Pitkanen et al. 1999; Rahman et al. 1998; Venugopal et al. 2004). Some of these strains have been proposed for use in aquaculture, which has prompted concern regarding potential ecological consequences should such fish accidentally enter natural ecosystems (Reichhardt 2000; Stokstad 2002). Large changes in growth phenotype in transgenic fish are associated with many underlying cellular and organismal physiological and behavioural changes, some of which have the potential to alter survival and reproductive success (fitness) of such fish should they enter natural ecosystems. Understanding the phenotypic effects of the transgene, as well as how these changes may influence interactions with ecosystem components, is necessary when assessing risk to natural populations. Ecological effects on ecosystems of transgenic strains are anticipated to depend on many factors: (1) the probability of entry to the ecosystem (by escape from confinement, or by purposeful introduction), (2) the survival and reproductive success (fitness) of the escaped individuals as well as their naturally-reared offspring, and (3) the consequences arising from use of ecosystem resources or genetic disruptions affecting the adaptability of populations (Devlin and Donaldson 1992; Devlin et al. 2006; Kapuscinski et al. 2007a, b; Kapuscinski and Hallerman 1990; Tiedje et al. 1989). Relative phenotypic and fitness differences between transgenic fish, non-transgenic conspecifics, and other ecosystem members are strong determinants of the probability of initial persistence and subsequent spread of a transgene, and hence the magnitude of potential consequences. The present paper seeks to examine the theoretically evolution of fitness and consequences of transgenic strains in nature arising from interaction between the transgene and other modifier loci in the genome.

Empirical assessments of engineered fish often are conducted by comparing the progenitor non-transgenic strain with a well-defined, often inbred, transgenic strain that reproducibly display uniform physiological and behavioural phenotypes. Since potential fitness effects and consequences of a transgenic strain will be highly dependant on its phenotypic differences from wild-type and/or other ecosystem members, understanding the potential for the transgenic phenotype to vary is critically important to allow accurate estimates of risk. Phenotypic variation in a transgenic strain can arise from several factors, including environmental effects, developmental plasticity, or epigenetics (Bessey et al. 2004; Devlin et al. 2004b; Devlin and Donaldson 1992; Hails and Morley 2005; Sundström et al. 2007; Weichman and Chaillet 1997). Variation also can arise from genetic changes, including alterations in transgene structure or from background allelic differences among individuals. In most natural fish populations, significant quantitative genetic variation exists which can influence the growth rate among individuals and strains (e.g. Dickerson et al. 2005; Gjedrem 2000; e.g. McClelland et al. 2005; Smoker et al. 1994; Tymchuk et al. 2006; Tymchuk and Devlin 2005). Such background genetic differences have the potential to interact with transgenes and influence phenotype, an effect that has been described in laboratory studies with other animal and plant systems (Alatalo et al. 2008; Eisen et al. 1993; Eisen et al. 1995; Engler et al. 1991; Linder 2001; Metz et al. 2006; Nadeau 2001; Nielsen et al. 1995; Parisi et al. 2003; Robertson et al. 2002; Siewerdt et al. 1998; Siewerdt et al. 2000; Suzuki et al. 2002; Weng et al. 1995). In mice, modifiers of GH transgenes have been observed and have been found to be responsive to artificial selection and to undirected natural selection (in the laboratory) that can strongly modify phenotype (Chaudhry et al. 2008; Siewerdt et al. 2000). Some of these transgene modifiers have been genetically mapped and have been found to act through the pathway of the gene of interest, or alternatively to affect epigenetic processes such as gene silencing by methylation (Dragani et al. 2000; Engler et al. 1991; Kantachuvesiri et al. 1999; Valenza-Schaerly et al. 2001). In transgenic fish, a transgene overexpressing growth hormone $(\mathrm{GH})$ has been found to have very different effects depending on genetic background. For example, in coho salmon, the GH transgene was found to work synergistically with a domesticated genome to enhance growth, whereas in rainbow trout, a wild (slow-growing) strain was found to be stimulated much more by the transgene than was a domesticated strain previously selected for very rapid growth (Devlin et al. 2001). 
Transcript profiling and endocrinology studies of GH transgenic and domesticated strains have shown that both influence gene expression patterns in similar ways relative to wild type (Devlin et al. 2009; Fleming et al. 2002; Raven et al. 2008; Tymchuk et al. 2009). Together, these observations reveal how a GH transgene can function differently among genetic backgrounds in vertebrates, and can have reduced influence on growth if previous traditional genetic selection of natural genetic variation has already exploited much of the potential opportunity to modify growth pathways and phenotype (Kitami and Nadeau 2002).

To facilitate assessments of potential ecological risks of transgenic fish, several fitness-based population models incorporating life history characteristics and ecological parameters have been developed. Modeling approaches have provided extremely valuable tools for examining the range of fitness parameter values that are expected to influence the persistence of a transgene in populations (Davis and Fulford 1999; Davis et al. 1999; Hedrick 2001; Maclean and Laight 2000; Muir and Howard 1999, 2001; Muir and Howard 2002). Several of these studies (Aikio et al. 2008; Davis and Fulford 1999; Maclean and Laight 2000) have identified the importance of initial frequency, repeat introductions and relative differences in fitness of transgenic and wild-type fish. Muir and Howard (1999, 2001, 2002) have further outlined major survival and reproductive fitness parameters anticipated to affect transgene persistence and population size. Their simulations also have shown that a transgene causing antagonistic pleiotropic effects (e.g., enhanced mating success, with reduced viability) could invade a population under specific conditions (Hedrick 2001), sweep into a population, and cause subsequent decline or extinction of the population, an outcome termed a Trojan gene effect (Muir and Howard 1999, 2001, 2002). These hypothetical scenarios advanced our thinking significantly regarding modeling risk of transgenic organisms, and recently Aikio et al. (2008) have shown further that densitydependence influences (which commonly act in fish populations) can overcome Trojan-gene effects and prevent population extinctions. Other modeling (Davis et al. 1999) has revealed that including stochasticity in survival and birthrate influences the movement of a transgene into populations (e.g., higher variance yields faster transgene introgression), and Valosaari et al.
(2008) have modeled the effect of mating success and age of maturation (within an evolutionarily stable strategy) on transgene frequencies in populations.

Previous modeling efforts have defined transgenic organisms as genetically and phenotypically uniform, and have not incorporated effects of background genetic variation that could result in the evolution of altered phenotype. Given the evidence that transgene modifier loci exist among laboratory strains, it is reasonable to assume that in nature, a transgene is very likely to encounter a range of genetic variation different from that found within the engineered strain (Devlin and Donaldson 1992; Devlin et al. 2007; Kapuscinski et al. 2007a). Over generations, the average phenotype caused by the transgene could evolve as selection acts on background alleles to improve fitness of individuals and populations. Such changes could influence consequences to ecosystems from that predicted from assessments made in the laboratory using a single strain with a relatively inbred genetic background. To examine these influences and extend previous models, we have undertaken an exercise to explore the potential for background genetic variation to influence transgene persistence in, and effects upon, populations. The present study has focussed on GH-transgenic coho salmon (Oncorhynchus kisutch) (Devlin et al. 2004a), which have been used as a model for transgenic fish risk assessment research (Devlin et al. 2006; Sundström et al. 2007). The theoretical population model developed allows background genetics (2-locus, or polygenic) to partially or fully modify the survival and reproductive fitness of transgenic and non-transgenic individuals through multiple overlapping generations in simulated populations. Our results indicate that background genetics has the potential to play an important role in mediating the persistence of transgenes in populations, and that evolutionary shifts in these populations may affect non-transgenic conspecifics as well.

\section{Methods}

Modelling effects of fitness parameters and background genetics on persistence of $\mathrm{GH}$ transgenic salmon in populations

A deterministic, age-structured model was developed to explore the theoretical effects of an introduction of 
GH-transgenic individuals into a wild salmonid population. The approach is similar to that previously developed by Muir and Howard $(1999,2001)$ except the present model focuses on salmonid life histories, incorporates potential effects of density-dependent juvenile survival, and includes effects of selection on background genetics (2-locus and polygenic) for multiple fitness parameters. For the 2-locus model, numbers of individuals and genotype frequencies over time were simulated assuming diploidy, with one modifier and one transgene locus (potentially 10 genotypes). One locus determines presence (transgene) or absence (wild type) of the transgene $\left(\mathrm{A}_{\mathrm{T}}\right.$ or $\mathrm{A}$, respectively), with the transgene having a dominant phenotypic effect $\left(\mathrm{A}_{\mathrm{T}} \mathrm{A}=\mathrm{A}_{\mathrm{T}} \mathrm{A}_{\mathrm{T}}\right)$. The second locus can act to modify (positively or negatively) fitness parameter values both for transgenic and wildtype genotypes (alleles $\mathrm{B}$ or $\mathrm{B}_{\mathrm{M}}$; the form $\mathrm{B}_{\mathrm{M}}$ hereafter referred to as the 'modifier'). The effects of the modifier were assumed to be additive, with $B_{M} B_{M}$ causing the greatest compensatory epistasis. Initial frequency of the $\mathrm{B}_{\mathrm{M}}$ polymorphism was arbitrarily assumed to be present in $10 \%$ of wild-type individuals.

Allowing for genotypic variability in female (j) fecundity $\left(F_{j}\right)$, male $(k)$ mating $\operatorname{success}\left(m_{k}\right)$, genotype $(i)$-specific survival through the first year of life $\left(s_{i, t}^{j}\right)$, and assuming Mendelian segregation $\left(M_{i, j, k}^{r}\right)$ under a given recombination rate $r$, expected numbers of offspring of genotype $i$ within the first age-class $\left(N_{i, t}^{j}\right)$ was modeled as

$N_{i, t}^{j}=s_{i, t-1}^{j} \sum_{j=1}^{10} \sum_{k=1}^{10}\left(p_{k, t-1} F_{j} N_{j, t-1}^{f} M_{i, j, k}^{r}\right)$.

The probability of a male $(l)$ mating $\left(p_{l, t}\right)$ depended on male mating success $\left(m_{k}\right)$ and male abundance $\left(N_{k, t}^{m}\right)$ such that

$p_{l, t}=m_{l} N_{l, t}^{m}\left(\sum_{k=1}^{10} m_{k} N_{k, t}^{m}\right)^{-1}$.

Male mating success was expressed relative to wildtype mating success. Juvenile survival $\left(s_{i, t}^{j}\right)$ in any year $(t)$ depended on total egg deposition $\left(E_{t}\right)$, and was modeled assuming that age-one recruitment followed the common Beverton-Holt form of recruitment relationship. Allowing for genotype-specific relative viability $\left(V_{i}\right)$, a maximum wild-type survival rate $(\alpha)$, and a parameter $(\beta)$ that represent densitydependent effects, juvenile survival was modeled as

$s_{i, t}^{j}=\alpha V_{i}\left(1+\beta E_{t}\right)^{-1}$

Maximum survival rate of wild-type juveniles $(\alpha)$ was parameterized as

$\alpha=\Omega \phi_{e} \quad$ where $\phi_{e}=0.5 F_{w} s^{A}\left(\varepsilon_{w}+\left(1-\varepsilon_{w}\right) s^{J 2}\right)$.

In this parameterization, $\Omega$ is the relative improvement in juvenile survival at low egg deposition levels from the juvenile survival expected under undisturbed conditions. $\varphi_{\mathrm{e}}$ is the expected lifetime egg production of a wild-type individual and depended on wild-type female fecundity $\left(F_{w}\right)$, the proportion of the population smolting after 1 year in fresh water $\left(\varepsilon_{w}\right)$ and the survival of smolts to return as adults the following year $\left(s^{A}\right)$, was assumed constant within a particular scenario. The proportion of juveniles that did not smolt after 1 year $\left(1-\varepsilon_{w}\right)$ was subjected to an additional survival adjustment $\left(S^{\mathrm{J} 2}\right)$ to account for additional fresh water losses. These individuals then survived at $s^{A}$ to account for smolt-to-adult losses. The $\beta$ parameter can be also represented in terms of $\Omega, \varphi_{\mathrm{e}}$, and maximum smolt production $\left(R_{0}\right)$ such that

$\beta=(\Omega-1)\left(R_{0} \phi_{e}\right)^{-1}$.

This parameterization is useful because juvenile production can be expressed in terms of life history characteristics, maximum juvenile production, and the relative strength of compensatory response in juvenile survival. For the simulation presented, female fecundity was assumed to be 4,000 eggs, smolt-to-adult survival was assumed $20 \%, S^{\mathrm{J} 2}$ was assumed to be $50 \%$, and the proportion of wild-type individuals smolting after 1 year was $10 \%$. As a result, maximum egg to age-one survival, assuming weak compensation $(\Omega=2)$, was around $1 \%$; with $\Omega=5$, survival increased to $2.2 \%$. Relative genotype-specific juvenile viability $\left(V_{i}\right)$ was assumed constant across all densities and potentially reduced for transgenic individuals. Females and males were assumed to spend 1 or 2 winters in freshwater, depending on genotype, and one winter in the ocean. Adult returns of a particular genotype and sex in a given year $\left(N_{i, t}^{f \text { or } m}\right)$ were modeled as 
$N_{i, t}^{f \text { or } m}=\varepsilon_{i} 0.5 N_{i, t-1}^{J} s^{A}+\left(1-\varepsilon_{i}\right) 0.5 N_{i, t-2}^{J} s^{J 2} s^{A}$.

Juvenile sex ratio was assumed to be 50:50, and the proportion of juveniles smolting after 1 year $\left(\varepsilon_{i}\right)$ was dependent on genotype.

For a number of simulations, the effects of the transgene were assumed to be influenced by multiple loci. In these polygenic scenarios, selection for favourable backgrounds was modeled as a progressive change in the transgenic phenotype toward the wild type. For the simulations presented in this paper it was assumed that juvenile viability, male mating advantage, and the proportion of juveniles smolting after 1 year had all changed relative to wild type. Equation 7 is an example of the calculation applied to viability:

$V_{T, g+1}=V_{T, g}+\vartheta\left(V_{w}-V_{T, g}\right)$.

Here, $V_{T, g}$ is the transgenic viability in generation $(g)$, $V_{W}$ is the wild-type vulnerability, and $\vartheta$ is the proportion of the difference between wild-type and transgenic viability reverted each generation. Thus, transgenic individuals asymptotically approach the wild type in terms of juvenile viability, male mating advantage, and the proportion of juveniles smolting after 1 year.

Density-dependant controls affecting survival were also present in the model. Relative change in equilibrium population size can be calculated using Eq. 8 for scenarios that resulted in transgene fixation. Under scenarios where the transgene reached fixation, relative change in population size was described using Eq. 9, and Eq. 10 defined the lower bound of $\Omega$.

$\frac{N_{e q}^{T}}{N_{e q}^{W}}=\frac{\left(V_{T} \Omega \phi_{e}^{T}-\phi_{e}^{W}\right)\left(-\varepsilon_{T}-S^{J 2}+S^{J 2} \varepsilon_{T}\right)}{\phi_{e}^{T}(\Omega-1)\left(-\varepsilon_{T}-S^{J 2}+S^{J 2} \varepsilon_{W}\right)}$

$\frac{N_{e q}^{T}}{N_{e q}^{W}}=\frac{V_{T}\left(-\varepsilon_{T}-S^{J 2}+S^{J 2} \varepsilon_{T}\right)}{\left(-\varepsilon_{W}-S^{J 2}+S^{J 2} \varepsilon_{W}\right)}$

$\Omega_{c}=\phi_{e}^{W} / V_{T} \phi_{e}^{T}$

A number of assumptions and simplifications are inherent in the model in order to facilitate description of the background genetic effects. As in Muir and Howard (1999) and Hedrick (2001), we have assumed a negative pleiotropic effect of the transgene that increases mating advantage in males, and reduces viability over the first year in freshwater for both sexes. This scenario allows for more complex analysis of interacting fitness components than if transgenes possess simply elevated or reduced net fitness. All reproductive females were assumed to mate and to have equal fecundity. Rapid growth of transgenic salmon may allow precocious smolt transformation (Devlin et al. 1994, 2000; Saunders et al. 1998), and thus $90 \%$ of transgenic individuals were assumed to smolt after their first year compared to $10 \%$ for the wild type. It is important to note, however, that the true development of transgenic fish in nature is suspected to be very different (i.e. slower) from that observed under hatchery conditions (Sundström et al. 2007). The relative increase in egg to first-year survival $(\Omega)$ was assumed similar for all genotypes regardless of the viability reduction caused by the transgene. For all simulations, population abundance was initialized at equilibrium given a maximum production of wild-type juveniles by the end of their first year in freshwater $\left(R_{0}=2 \times 10^{5}\right)$. Introduction of transgenic individuals $\left(\mathrm{A}_{\mathrm{T}} \mathrm{B} \mathrm{A}_{\mathrm{T}} \mathrm{B}\right)$ was simulated as a single event with an abundance of $0.1 \%$ of the equilibrium wild-type spawning population $\left(N_{O}\right)$.

The model also allowed for varying the linkage relationship between the modifier locus and the transgene, from tight linkage ( $0 \%$ recombination) to distant linkage (50\% recombination). However, there was little effect unless the modifier was tightly linked in cis (for example mimicking a structural change at the transgene locus). Thus, scenarios were modeled with modifiers in trans and unlinked (most modifiers are anticipated to in reality be in this state relative to the transgene).

\section{Results}

Potential effects of altered fitness parameters and life history traits on transgene frequency and population size in $\mathrm{GH}$ transgenic salmon

Base simulations with the model (without action of modifiers) yielded results similar to those previously described (Davis and Fulford 1999; Davis et al. 1999; Hedrick 2001; Maclean and Laight 2000; Muir and Howard 1999, 2001; Muir and Howard 2002). The model also effectively approximated salmon population dynamics, responding as expected to variation in survival, age of maturation, reproductive fitness 


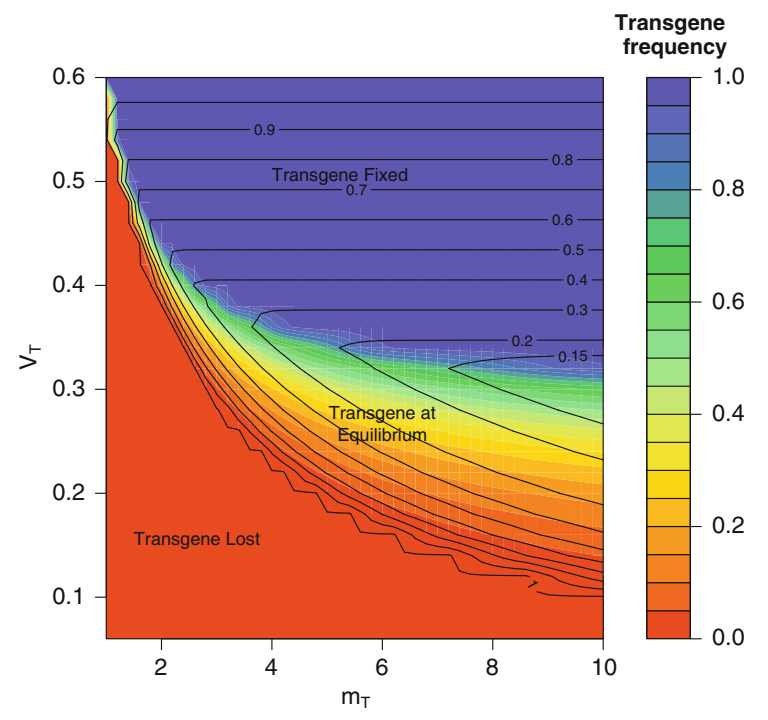

Fig. 1 Equilibrium spawning population relative to the pure wild-type equilibrium population size $\left(N_{e q}^{T} / N_{e q}^{W}\right.$, black contour lines) and transgene frequency $\left(\mathrm{A}_{\mathrm{T}}\right)$ (colour shaded contours) as a function of relative transgenic age-1 viability $\left(V_{T}\right)$ and transgenic male mating advantage $\left(m_{T}\right)$ assuming no effect of background genetics on the transgene. Viability and mating advantage were scaled relative to wild type. Darkest blue shading indicates fixation of the transgene while dark orange indicates the transgene is lost. $90 \%$ of transgenic juveniles were assumed to smolt after 1 year in this scenario, while only $10 \%$ of wild juveniles smolted after 1 year. Relative improvement in juvenile survival at low density was set low $(\Omega=2)$

parameters, and life history variation. The scenario depicted in Fig. 1 shows that when fitness components with opposing effects (enhanced male mating success, coupled with reduced survival as first year juveniles and in the smolt to adult interval) are combined, the transgene is able to invade the population over a wide range of conditions, reaching either fixation or a stable equilibrium. The presence of the transgene also can reduce mean population fitness and result in lower equilibrium population size $\left(N_{e q}^{T}\right)$ compared to a wildtype $\left(N_{e q}^{W}\right)$ population $\left(N_{e q}^{T} / N_{e q}^{W}<1\right.$, i.e., a 'Trojan gene' effect). Relative change in population size (Eq. 8) depends on juvenile survival rate reduction for individuals possessing the transgene $\left(V_{T}\right)$ as well as the strength of density-dependent compensatory changes in juvenile survival $(\Omega$; Fig. 2 a). Expected lifetime fecundity of GH-transgenic $\left(\phi_{e}^{T}\right)$ and wildtype $\left(\phi_{e}^{W}\right)$ individuals, survival during the second year of freshwater residence, and the proportion of individuals smolting after 1 year of freshwater residence $\left(\varepsilon_{W}\right.$ or $\left.\varepsilon_{T}\right)$, also can strongly influence changes in allele frequency and population size. It is important to note that in simulations where a high proportion of transgenic individuals matured at only 2 years of age and the juvenile survival reduction associated with the transgene was weaker (i.e., $80 \%$ of wild-type), equilibrium population size increased even when male mating advantage was only twofold (solid grey line Fig. 2a). When viability effects increased (i.e. $50 \%$ of wild type) at the same mating advantage, population size did not increase and smaller reductions in population size were predicted relative to the more extreme scenario $\left(V_{T}=0.3\right.$ and $\left.m_{T}=5\right)$. Reduction in mean generation time of transgenic individuals caused transgene frequencies and relative equilibrium population size to increase, even when juvenile viability was reduced. Increased juvenile survival compensation $(\Omega)$ reduced the relative decline in population size. Under scenarios where the transgene reached fixation, relative change in population size approached an asymptote described by Eq. 9. Although compensatory changes in survival in response to reduced population density prevents population extinction under a 'Trojan gene' scenario, strong reductions in population size are still possible when density dependent effects on juvenile survival $(\Omega)$ are weak. Equation 10 defines the lower bound on $\Omega$ (Fig. 2b). Below these values of $\Omega$, under a 'Trojan gene' scenario, population extinction occurred. It is important to note that as the viability of transgenic individuals declines, the critical bound on $\Omega$ increases geometrically.

Potential effects of background genetics on transgene effects

We now consider how modification of the fitness of transgenic individuals due to variation in background genetics may influence the outcome of transgene introductions into natural populations. In many cases, background genetics would differentially affect various fitness parameters in transgenic individuals, for example by ameliorating disease susceptibility effects (e.g., Jhingan et al. 2003), but not affecting mating advantage or age of maturity. Thus, the model allows modifier effects to be applied differentially to individual fitness parameters. The effects of background genetics were modeled initially with a single modifier 


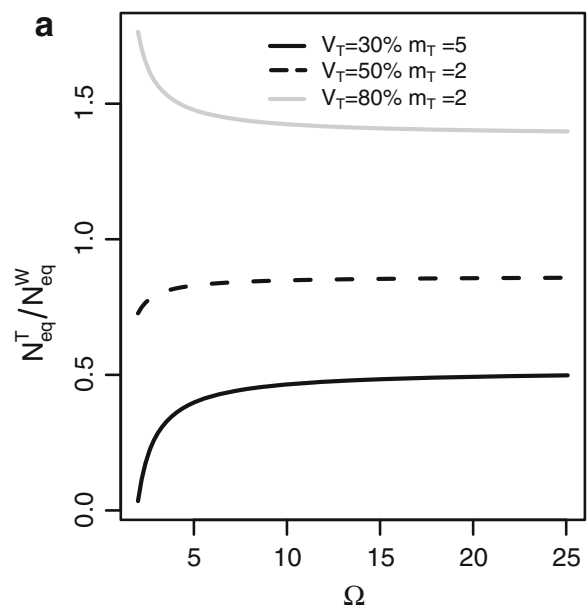

Fig. 2 Change in relative equilibrium population size as a function of the strength of compensatory change in juvenile survival $(\Omega)$ for a 'Trojan gene' scenario (Panel a black solid line) where transgenic viability was $30 \%\left(V_{T}=0.3\right)$ of wild type and the male mating advantage was 5 times $\left(m_{T}=5\right)$. This is contrasted with less extreme scenarios where transgenic viability was $80 \%\left(V_{T}=0.8\right)$ of wild type and the male mating advantage was 2 times $\left(m_{T}=2\right)$ (solid gray line) and where

locus present in the population that, in one allelic form $\left(B_{M}\right)$, counters the effect of the transgene on multiple fitness parameters. For the example shown (Fig. 3), the relative juvenile viability, mating advantage, and maturity schedules of individuals possessing the transgene with two copies of the modifier $\left(\mathrm{B}_{\mathrm{M}}\right)$ were assumed to be halfway between the wildtype and transgenic phenotypes. Individuals with only one copy of the modifier were assumed intermediate between transgenic individuals and those possessing two copies of the modifier. Given these compensatory epistatic effects of the modifier, the transgene reached fixation (blue area) over a broader range of scenarios (juvenile viability $\mathrm{x}$ mating advantage combinations), with the modifier reaching fixation (white hatched area) over a range of scenarios as well. Population levels with the modifier (Fig. 2) were depressed over much of the range examined, although the severity of impact was strongly reduced compared to scenarios lacking the modifier (Fig. 1). The range of scenarios under which the transgene was maintained at a stable equilibrium by frequency-dependent selection also was substantially compressed (compare the size of coloured zones between dark blue and dark red in Figs 1 and 3). Over a range of scenarios (white hatched area),

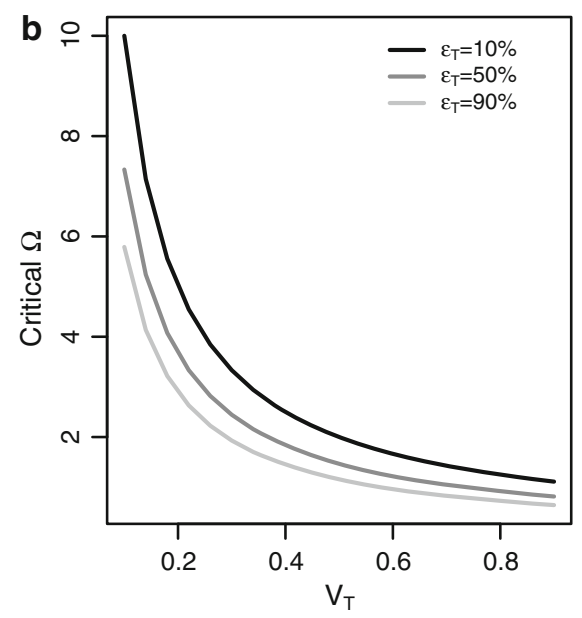

transgenic viability was $50 \%\left(V_{T}=0.5\right)$ of wild type and the male mating advantage was 2 times $\left(m_{T}=2\right)$ (broken black line). Panel $\mathbf{b}$ displays how the critical compensation ratio $(\Omega)$ changes as a function of viability differences in transgenic individuals $\left(V_{T}\right)$ and the proportion of transgenic individuals smolting after 1 year $\left(\varepsilon_{T}\right)$. Below the critical compensation ratio, a Trojan gene scenario resulted in population extinction

transgenic individuals with the modifier gained a fitness advantage, transgene spread was enhanced, and the modifier became fixed. This region of modifier fixation exhibits transitions into zones of scenarios where transgenic individuals with and without the modifier are in a stable equilibrium, with the modifier being maintained in the population through frequency-dependent selection. At higher levels of viability, transgenic individuals without the modifier can have a greater fitness advantage and the modifier is lost from the population. As the modifier spreads in the population, improved viability of transgenic individuals due to the presence of the modifier can ameliorate population declines (compare Figs $4 \mathrm{a}$ and $\mathrm{b}$ ). Although the severity of population impact is reduced under 'Trojan gene' scenarios, background variation that was neutral in the absence of the transgene experienced selection in the presence of the transgene. As a result, in the scenario presented, the modifier reached fixation (compare Figs $4 d$ and e). If the modifier fully ameliorated the effects of the transgene (Fig. 4c), the fitness advantage of the 'modifier-transgene' construct was reduced. As a result, population recovery was less and the modifier did not reach fixation. If a heterozygote advantage for non-transgenic individuals 


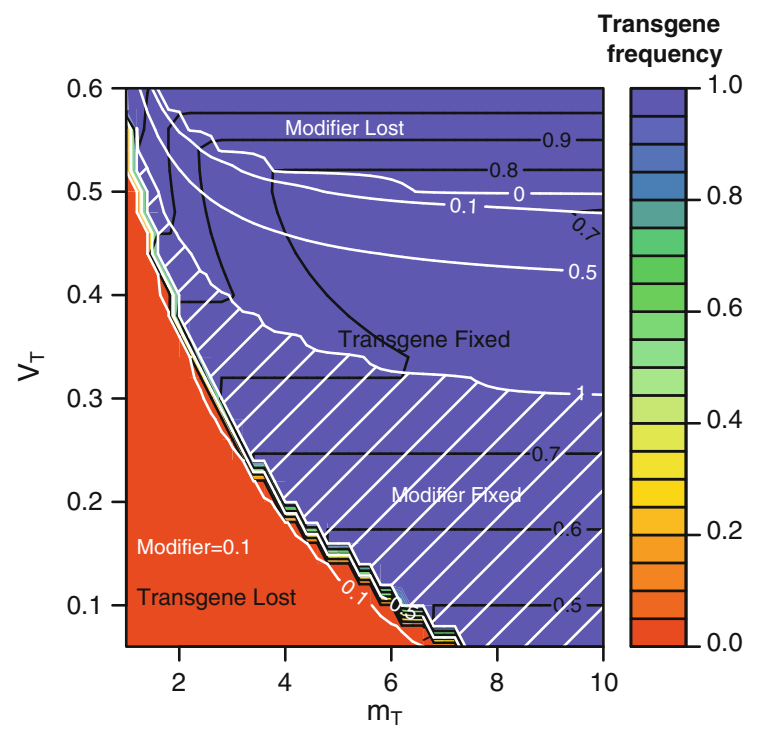

Fig. 3 Equilibrium relative spawning population size $N_{e q}^{T} / N_{e q}^{W}$ (black contour lines), transgene frequency $\left(\mathrm{A}_{\mathrm{T}}\right)$ (colour shaded contours) and modifier frequency $\left(\mathrm{B}_{\mathrm{M}}\right)$ (white contour lines and hatching) as a function of relative transgenic juvenile viability $\left(\mathrm{V}_{\mathrm{T}}\right)$ and transgenic male mating advantage $\left(m_{T}\right)$. Viability and mating advantage are scaled relative to wild type. Blue shading indicates fixation of the transgene $\left(p\left(\mathrm{~A}_{\mathrm{T}}\right)=1\right)$, while dark orange indicates that the transgene is lost $\left(p\left(\mathrm{~A}_{\mathrm{T}}\right)=0\right)$. Hatched white area indicates fixation of the modifier allele $\left(p\left(B_{M}\right)=1\right)$. Modifier $\left(B_{M}\right)$ frequency within the white area is at its initial frequency $(0.1)$. Relative equilibrium spawning population size $\left(N_{e q}^{T} / N_{e q}^{W}\right)$ is scaled relative to the equilibrium spawning population in the absence of the transgene. $90 \%$ of transgenic juveniles are assumed to smolt after 1 year in this scenario, while only $10 \%$ of wild-type juveniles smolt after 1 year. Relative improvement in juvenile survival at low density was set low $(\Omega=2)$

existed at the modifier locus, variation can be maintained by balancing selection. However, in the presence of a transgene, alleles that are unfavourable in homozygous form for wild type were selected and caused a more rapid decline in wild-type numbers.

When the interaction of many genes was assumed to ameliorate the effects of the transgene (Eq. 7), the dynamics of the population were somewhat different. In these polygenic scenarios, if selection for favourable backgrounds was low (Fig. 4g), the transgene reached fixation and the reduction in population abundance due to 'Trojan gene' effects was reduced. On the other hand, if selection was rapid (Fig. 4h), and reversion of the transgenic phenotype occurred before the transgene reached fixation, the transgenic genotype becomes a stable polymorphism.
The simulation results presented so far have all assumed that a high proportion $(90 \%)$ of transgenic individuals smolt after 1 year and mature in 2 years as is seen for GH transgenic coho salmon under laboratory conditions (Devlin et al. 2004b). The relative maturity schedules affect both the range of viability reductions and mating advantages over which the transgene introgressed as well as the equilibrium population size. When transgenic individuals are assumed to mature on a wild-type schedule (e.g. 3 years), a 'Trojan gene' effect was observed at lower mating advantages and at smaller viability reductions (Fig. 4i). When a high proportion of transgenics were assumed to mature at age $2(90 \%)$ a 'Trojan gene' effect is not seen and the population equilibrium size is higher than with wild-type alone. Changing the maturity schedule of transgene fish effectively shifts the zone described in Fig. 1 upwards.

\section{Discussion}

The present modeling exercise, using a growthenhanced coho salmon as a theoretical example, examined the importance of background genetic effects in modifying the outcome of transgene introductions into populations. The model allows examination of the effect of selection for background genetic alleles that partially or fully overcome negative fitness traits associated with a transgene. When genetic backgrounds ameliorate the effects of the transgene, it was found that the range of conditions over which the transgene can invade a population was increased, and that the transgene can be maintained in equilibrium by frequency-dependant selection under some conditions. Under circumstances where a transgene would otherwise be lost, novel genetic backgrounds can facilitate persistence of the transgene over a broader range of conditions if selection for favourable backgrounds occurs at a rate greater than the inherent loss rate for the transgene. Thus, modifier alleles causing fitness impairments in the wild-type that counter-select the transgene phenotypes are anticipated to increase in frequency in populations and influence ecological consequences arising from transgenic individuals.

Growth rate in fish is a continuous quantitative character that is polygenically influenced by (mainly) additive genetic variance (e.g., Gjedrem 2000; 

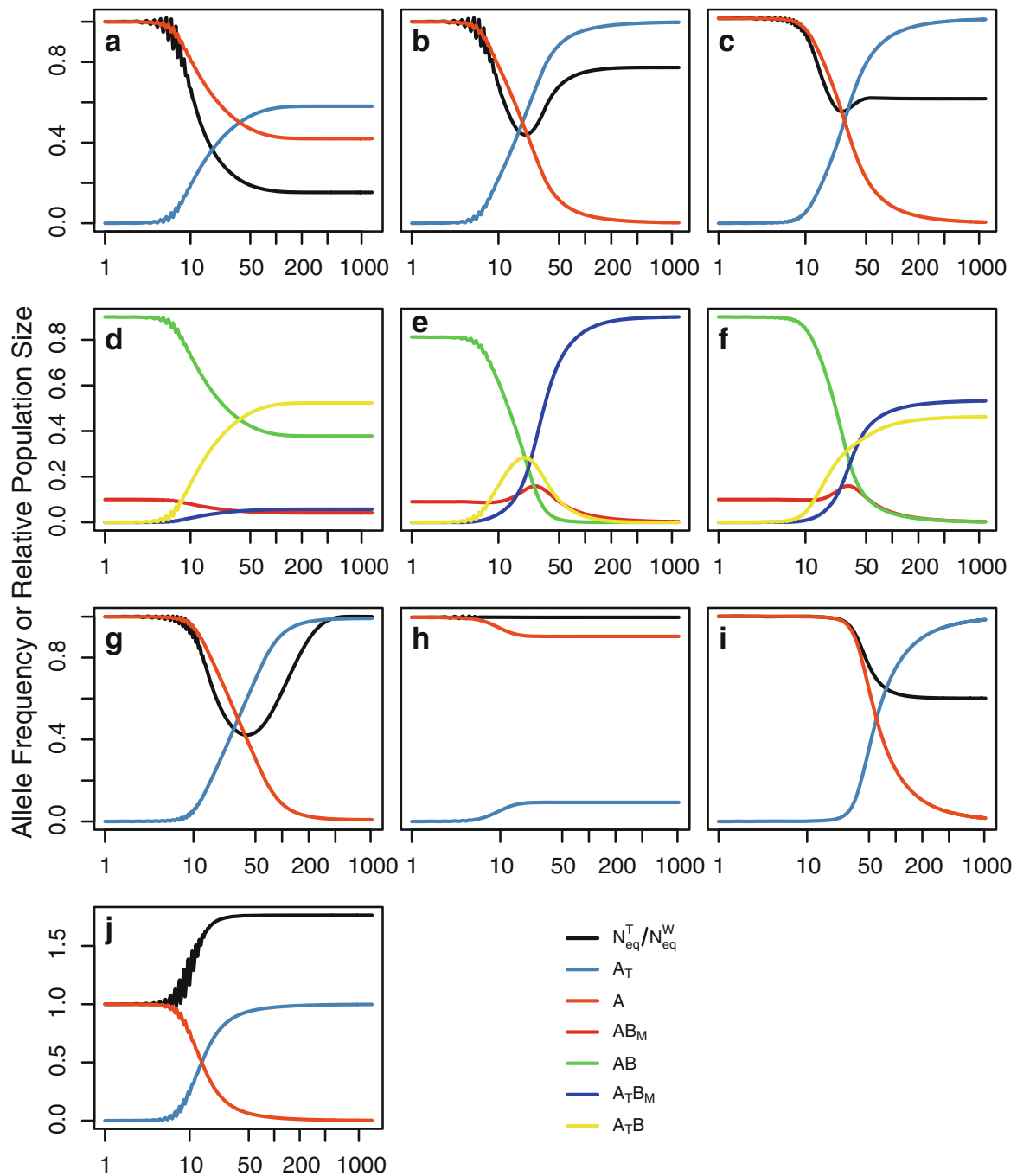

Generations

Fig. 4 Equilibrium population sizes and gene frequencies from 'Trojan gene' scenarios where unmodified transgene viability was set to 0.3 and transgenic mating advantage was set at 5. Relative improvement in juvenile survival at low density was set low $(\Omega=2)$. Panels a and $\mathbf{d}$ represent relative population and gene frequency changes in the absence of the modifier, and panels $\mathbf{b}$ and $\mathbf{e}$, as well as $\mathbf{c}$ and $\mathbf{f}$, represent these changes in the presence of the modifier. In panels $\mathbf{b}$ and $\mathbf{e}$, the 'modifier-transgene' phenotype is half way between the wildtype and transgenic phenotypes, and in $\mathbf{c}$ and $\mathbf{e}$ the 'modifiertransgene' phenotype is equal to the wild type. Panels $\mathbf{g}$ and $\mathbf{h}$ are from polygenic scenarios where selection intensity was assumed to be low $\vartheta=0.002(d)$ or high $\vartheta=0.1(h)$. Panels $\mathbf{i}$ and $\mathbf{j}$ are provided for contrast. In panel $\mathbf{i}$, transgenic viability and mating advantage are set to 0.8 and 2 , respectively, but transgenic individuals smolt and mature at the same time as wild type. In panel $\mathbf{j}$, transgenic viability and mating advantage are set to 0.8 and 2 respectively but transgenic individuals do smolt and mature 1 year earlier than wild type. In panels ac and $\mathbf{g}-\mathbf{j}$, the black line indicates $N_{e q}^{T} / N_{e q}^{W}$, the red line is the frequency of wild-type genotype, and the blue line transgenic genotype. In panels $\mathbf{d}-\mathbf{f}$, changes of the genotypes are represented: $\mathrm{AB}_{\mathrm{M}}$ the wild type with the modifier (red line) and $\mathrm{AB}$ without the modifier (green line). The blue and yellow lines are the frequencies of the transgene with $\left(\mathrm{A}_{\mathrm{T}} \mathrm{B}_{\mathrm{M}}\right)$ and without $\left(\mathrm{A}_{\mathrm{T}} \mathrm{B}\right)$ the modifier, respectively. The initial modifier frequency was set to 0.1 . $90 \%$ of transgenic juveniles were assumed to smolt after 1 year in all scenario scenarios but I, while only $10 \%$ of wild juveniles smolted after 1 year 
McClelland et al. 2005; Tymchuk et al. 2006; Tymchuk and Devlin 2005) and is associated with reasonably high heritabilities and responses to selection (Dickerson et al. 2005; Gall and Huang 1988; Gjedrem 2000; Hershberger et al. 1990; Smoker et al. 1994). Wild fish have long been selected for optimal growth rate, but for many species, growth rates in nature are below that which is physiologically possible. For example, salmonids are capable of being strongly growth stimulated through environmental, nutritional, or hormonal manipulations (Ali et al. 2003; Donaldson et al. 1979), suggesting that less than maximal growth rates are adaptive in nature and arise from fitness tradeoffs with other components of Darwinian fitness, for example, involving traits pertaining to physiological requirements (such as disease resistance), or to minimize predation risk from foraging (Arendt 1997; Lima and Dill 1990). Indeed, elevation of growth rate away from wild type (e.g. in GH transgenic or domesticated salmon) can result in increased risk taking and reduced fitness via increased predation mortality (Abrahams and Pratt 2000; Abrahams and Sutterlin 1999; Biro et al. 2004; Sundström et al. 2004, 2005; Tymchuk et al. 2005; Tymchuk et al. 2006; Tymchuk et al. 2007). Thus, background genetic variation that reduces growth rate of transgenic fish back towards the natural fitness optimum probably would be selectively favoured in natural populations. Further, observed pleiotropic effects of GH transgenesis on morphology, physiology, and behaviour could have negative fitness consequences (e.g. Bessey et al. 2004; Deitch et al. 2006; e.g. Farrell et al. 1997; Leggatt et al. 2003; Ostenfeld et al. 1998; Stevens and Devlin 2000; Sundt-Hansen et al. 2007), and selection at modifier loci that compensate for these specific effects would be anticipated. For example, disease impairments in GH transgenic salmon (Jhingan et al. 2003) might be overcome by selection at immune function loci, and could do so without altering the enhanced growth rate. Thus, modifiers are expected to be prevalent in the genome and of multiple types, affecting growth generally or targeting specific pleiotropic phenotypes caused by the transgene.

Population size effects arising from transgenemodifier interactions were apparent in the present study in both the two-locus and polygenic simulations. The present salmon model has predicted Trojan gene effects on population size (e.g., as shown by Hedrick 2001; Muir and Howard 1999, 2001) under specific conditions, but these consequences could be ameliorated to a large degree (2 locus model) or completely (polygenic) by selection occurring at modifier loci. As such, transgene-countering loci would be anticipated to be selected for in nature, thereby restoring population level fitness. In an analogous system in nature (segregation distortion, $\mathrm{SD}$ ), meiotic drive can cause chromosomes with SD alleles to preferentially segregate into populations, which, if sex linked, can skew sex ratios and cause population declines (Hamilton 1967). Thus, it is not surprising that extant populations containing SD alleles also possess modifiers that counter these effects (Jaenike 2001). Huang et al. (2007) modeled mosquito-control transgenes being driven into populations by a meiotic drive system, and have found that the presence of unlinked genetic modifiers can strongly influence the spread of the transgene. In the present model, in addition to population reduction effects, we also observed scenarios where genetic modifiers acted in concert with the transgene to cause population increase beyond those seen with wild type alone. This outcome might occur, for example, if fitness 'valleys' could be bridged to allow development of novel phenotypes unachievable by populations using extant natural allelic variation, or because of strong stabilizing selection.

In addition to genetic compensatory responses arising from modifier-transgene interactions, the present model also predicted that demographic controls can strongly dampen population effects associated with transgene-induced Trojan gene scenarios. Consistent with the findings of Aikio et al. (2008), density-dependent effects causing increased survival at lower population sizes are capable of minimizing negative fitness effects of transgenes. Reductions in population size can still ensue from antagonistic pleiotropic effects even in the presence of compensatory responses; however, for the scenario presented herein mimicking the salmon life history, very large increases in mating advantage of transgenic fish over wild type were required to induce strong depressions in population size. Further, none of the scenarios we examined predicted population extinctions, even when mating advantage was 10 -fold greater than wild type (a biologically unrealistic value).

Shifts in life-history characteristics caused by growth enhancement may prove critical in influencing transgene effects in populations. For example, 
GH-transgenic coho salmon have been shown to develop more rapidly embryologically and to emerge sooner from natal gravel redds, reducing their fitness by increasing their susceptibility to predation mortality (Devlin et al. 2004a; Sundström et al. 2005). GH-transgenic salmon also can develop faster through their freshwater phase, undergoing early smoltification (the physiological adaptation and migration from a fresh water to marine environment) in their first rather than second year of life (Devlin et al. 1994, 2000; Saunders et al. 1998). Thus, the timing of smolt migration is expected to influence the location where ecological consequences will occur (e.g., resource use in stream vs. marine environments), and would also affect the level of mortality experienced by transgenic individuals. Consistent with this, the present model predicts that transgene frequency in populations depends on the duration that transgenic fish remain in freshwater. Since survival improvement by early smoltification can offset viability reductions caused by the transgene phenotype at other stages, overall fitness of the transgenic phenotype can be enhanced. Evolution of such pleiotropic effects of the transgene and their interactions with the environment via selection of modifiers needs to be understood to facilitate accurate predictions of natural fitness and potential ecological consequences.

GH-transgenesis also can influence maturation age and adult body size in fish. For example, GHtransgenic coho salmon mature with a body size similar to wild type, but do so at 2 years rather than 4 years of age for wild-type fish reared in the laboratory (or 3 years for wild coho salmon) (Devlin et al. 1995, 2004a). In contrast, adult GH-transgenic rainbow trout mature at their normal age of 3 years, but at a much larger size (remarkably, 37- to 83-fold heavier) than their wild-type counterparts (Devlin et al. 2001). Larger adult fish (particularly male salmonids) are known to possess a breeding advantage in several salmonid species (Fleming 1998), prompting the suggestion that GH-transgenic fish may have a mating advantage facilitating the spread of the transgene. In medaka (Oryzias latipes), larger $\mathrm{GH}$-transgenic males have been found to possess a mating advantage over wild-type males raised under the same conditions (Howard et al. 2004), providing support that GH-transgenic fish possess a reproductive fitness advantage. In GH-transgenic coho salmon, spawning ability was found to be inferior to wild type in laboratory trials in simulated streams (Bessey et al. 2004). However, because non-transgenic salmon reared in the same laboratory environment also showed impaired reproductive success, it was difficult to accurately partition genetic (transgene vs. wild type) and environmental (laboratory vs. nature) contributions to effects on breeding capabilities. Reproduction involves an extremely complex and flexible set of evolved morphologies, behaviours and physiologies, and indeed reproductive success in fish is not always associated with large body size. Several salmonid species possess an evolutionarily stable strategy where fast-growing males can reach maturation 'precociously' (at an age and size less than adult females) and employ a 'sneaker' or 'jack' strategy to fertilize eggs in spawning events involving regular males and females (Groot and Margolis 1991; Gross 1991). Such males transmit their genetic traits through generations faster than do regular males that mature at an older age. Thus, reductions in the age of maturation by $\mathrm{GH}$-transgenesis has the potential to facilitate more rapid generational transmission of the transgene allele relative to other genetic variation in the population (Muir and Howard 1999, 2001). Indeed, Valosaari et al. (2008) have modeled how this evolutionarily stable mating strategy (i.e., precocious male maturation) in Atlantic salmon could modify effects GH-transgenic fish on populations and transgene persistence. Although GH-transgenic coho salmon do not show precocious maturation relative to transgenic females, both sexes do mature earlier than wild type. As expected, this compression of their life history has been predicted with the present model to facilitate transgene spread, even in the absence of other fitness advantages relative to wild type. Further, early maturation of transgenic fish at 2 years of age predicts elevated population levels under conditions (e.g. $V_{T}=0.8 ; m_{T}=2$ ) that otherwise cause a Trojan gene decline when transgenic fish mature at the normal 3 years of age.

Selection acting on transgenic individuals affecting the type and frequency of background genetic variation in populations has the potential to influence the phenotype of non-transgenic conspecifics as well. Loci that influence traits affected by the transgene are likely abundant in most natural populations, and variation at such loci would be under continual selection (directional and stabilizing) across the range 
of fluctuating environments and selection regimes experienced by the organism. The entry of a transgene into a population could redirect selection upon this variation and consequent phenotypes away from that normally affording maximal fitness in wild-type individuals. The present model predicted that a modifier under selection by enhancing the fitness of transgenic fish can also affect non-transgenic populations that were otherwise maintaining variation at the modifier locus via balancing selection (e.g., via heterozygote advantage or from alternating directional selection in response to fluctuating environmental conditions). The presence of a transgene in a population could create a situation where selection of background genetic variation would be occurring counter to that favoured in wild-type individuals. This polarized selection could place a genetic load (Haldane 1957) on all individuals in the population by reducing the probability they would possess optimally selected background genetic variation for their genotype (transgenic or wild type). If counter selection of non-optimal background alleles does not occur rapidly in non-transgenic individuals, their suboptimal genotypes could further facilitate persistence of the transgene. In population fitness studies with Tribolium, a novel (eye-colour) allele was observed to cause higher individual fitness and therefore increased in frequency in one replicate population, but at the same time was associated with an overall decline in population size (Dawson 1969). This effect was attributed to a reproductive load arising in response to a "reshuffling of the gene pool" (e.g., selection of optimal background genetic variation) while adaption to the presence of the novel mutant allele occurred.

The simulation model presented here and those developed previously by other authors provide tools for understanding critical variables that play roles in influencing the persistence of transgenes in nature. However, we caution that using these theoretical constructs as tools to predict outcomes in nature is likely associated with a high level of uncertainty. For salmonids, the models developed to date, including the present one, do not fully reflect the complexities of their life histories, and do not incorporate the powerful environmental fluctuations that can be strong determinants of population size in salmonids. Further, ascribing parameter values that truly reflect those which would exist in nature cannot easily be validated based on the extant laboratory data alone.
Complex pleiotropic effects of a GH-transgene are apparent across the full life history, and many of these phenotypic changes are strongly plastic and show non-parallel reaction norms across environments (Bessey et al. 2004; Devlin et al. 2004b; Sundström et al. 2005; Sundström et al. 2007). For risk assessments of fertile, highly-viable strains of transgenic salmon, comprehensive data sets from a broad range of conditions and life histories stages would be beneficial for prediction of potential consequences to ecosystems utilized by the species. The present modeling exercise has shown that understanding how transgene effects may evolve over time also will be critical for robust predictions of potential ecological consequences, and hence risk.

Acknowledgments The authors thank Sally Otto for helpful discussions regarding details of the model structure, and acknowledge support from the Canadian Regulatory System for Biotechnology to RHD. We also thank two anonymous reviewers for their helpful comments and suggestions.

Open Access This article is distributed under the terms of the Creative Commons Attribution Noncommercial License which permits any noncommercial use, distribution, and reproduction in any medium, provided the original author(s) and source are credited.

\section{References}

Abrahams MV, Pratt TC (2000) Hormonal manipulations of growth rate and its influence on predator avoidanceforaging trade-offs. Can J Zool 78:121-127

Abrahams MV, Sutterlin A (1999) The foraging and antipredator behaviour of growth-enhanced transgenic Atlantic salmon. Anim Behav 58:933-942

Aikio S, Valosaari KR, Kaitala V (2008) Mating preference in the invasion of growth enhanced fish. Oikos 117:406-414

Alatalo I, Haviola S, Saloniemi I (2008) Genetic background affects genetically modified ethylene sensing in birchinsect interaction. Botany-Botanique 86:988-994

Ali M, Nicieza A, Wootton RJ (2003) Compensatory growth in fishes: a response to growth depression. Fish Fish 4:147-190

Arendt JD (1997) Adaptive intrinsic growth rates: an integration across taxa. Quart Rev Biol 72:149-177

Bessey C, Devlin RH, Liley NR, Biagi CA (2004) Reproductive performance of growth-enhanced transgenic coho salmon (Oncorhynchus kisutch). Trans Amer Fish Soc 133:1205-1220

Biro PA, Abrahams MV, Post JR, Parkinson EA (2004) Predators select against high growth rates and risk-taking behaviour in domestic trout populations. Proc R Soc Lond B 271:2233-2237 
Chaudhry AM, Marsh-Rollo SE, Aksenov V, Rollo CD, Szechtman H (2008) Modifier selection by transgenes: the case of growth hormone transgenesis and hyperactive circling mice. Evol Biol 35:267-286

Davis S, Fulford G (1999) Modelling the integration of a transgene by stocking. Theor Popul Biol 55:53-60

Davis SA, Catchpole EA, Pech RP (1999) Models for the introgression of a transgene into a wild population within a stochastic environment, with applications to pest control. Ecol Model 119:267-275

Dawson PS (1969) A conflict between Darwinian fitness and population fitness in Tribolium competition experiments. Genetics 62:413-419

Deitch EJ, Fletcher GL, Petersen LH, Costa IASF, Shears MA, Driedzic WR, Gamperl AK (2006) Cardiorespiratory modifications, and limitations, in post-smolt growth hormone transgenic Atlantic salmon Salmo salar. J Exp Biol 209:1310-1325

Devlin RH, Donaldson EM (1992) Containment of genetically altered fish with emphasis on salmonids. In: Hew CL, Fletcher GL (eds) Transgenic fish. World Scientific Press, Singapore, pp 229-265

Devlin RH, Yesaki TY, Biagi CA, Donaldson EM, Swanson P, Chan W-K (1994) Extraordinary salmon growth. Nature 371:209-210

Devlin RH, Yesaki TY, Donaldson EM, Hew CL (1995) Transmission and phenotypic effects of an antifreeze/GH gene construct in coho salmon (Oncorhynchus kisutch). Aquaculture 137:161-170

Devlin RH, Swanson P, Clarke WC, Plisetskaya E, Dickhoff W, Moriyama S, Yesaki TY, Hew CL (2000) Seawater adaptability and hormone levels in growth-enhanced transgenic coho salmon, Oncorhynchus kisutch. Aquaculture 191:367-385

Devlin RH, Biagi CA, Yesaki TY, Smailus DE, Byatt JC (2001) Growth of domesticated transgenic fish. Nature 409:781-782

Devlin RH, Biagi CA, Yesaki TY (2004a) Growth, viability and genetic characteristics of GH transgenic coho salmon strains. Aquaculture 236:607-632

Devlin RH, D'Andrade M, Uh M, Biagi CA (2004b) Population effects of growth hormone transgenic coho salmon depend on food availability and genotype by environment interactions. Proc Nat Acad Sci USA 101:9303-9308

Devlin RH, Sundstrom LF, Muir WM (2006) Interface of biotechnology and ecology for environmental risk assessments of transgenic fish. Trends Biotechnol 24:89-97

Devlin RH, Sundström LF, Johnsson JI, Fleming IA, Hayes KR, Ojwang WO, Bambaradeniya C, Zakaraia-Ismail M (2007) Assessing ecological effects of transgenic fish prior to entry into nature. Chapter 6. In: Kapuscinski AR, Hayes KR, Li S, Dana G (eds) Environmental risk assessment of genetically modified organisms, volume 3: methodologies for transgenic fish. CABI International, Cambridge, pp 151-187

Devlin RH, Sakhrani D, Tymchuk WE, Rise ML, Goh B (2009) Domestication and growth hormone transgenesis cause similar changes in gene expression in coho salmon (Oncorhynchus kisutch). Proc Nat Acad Sci USA 106:3047-3052

Dickerson B, Wilson M, Bentzen P, Quinn T (2005) Heritability of life history and morphological traits in a wild pink salmon population assessed by DNA parentage analysis. Trans Am Fish Soc 134:1323-1328

Donaldson EM, Fagerlund UHM, Higgs DA, McBride JR (1979) Hormonal enhancement of growth. In: Hoar WS, Randall DJ, Brett JR (eds) Fish physiology: bioenergetics and growth. Academic Press, New York, pp 455-597

Dragani TA, Peissel B, Zanesi N, Aloisi A, Dai Y, Kato M, Suzuki H, Nakashima I (2000) Mapping of melanoma modifier loci in RET transgenic mice. Jpn J of Cancer Res 91:1142-1147

Du SJ, Gong Z, Fletcher GL, Shears MA, King MJ, Idler DR, Hew CL (1992) Growth enhancement in transgenic Atlantic salmon by use of an "all fish" chimeric growth hormone gene construct. Biotechnology 10:176-181

Eisen EJ, Fortman M, Chen WY, Kopchick JJ (1993) Effect of genetic background on growth of mice hemizygous for wild-type or dwarf mutated bovine growth hormone transgenes. Theor Appl Genet 87:161-169

Eisen EJ, Murray JD, Schmitt TJ (1995) An ovine-growthhormone transgene model suitable for selection experiments for growth in mice. $\mathrm{J}$ Anim Breeding Genet 112:401-413

Engler P, Haasch D, Pinkert CA, Doglio L, Glymour M, Brinster R, Storb U (1991) A strain-specific modifier on mouse chromosome-4 controls the methylation of independent transgene loci. Cell 65:939-947

Farrell AP, Bennett W, Devlin RH (1997) Growth-enhanced transgenic salmon can be inferior swimmers. Can J Zool 75:335-337

Fleming IA (1998) Pattern and variability in the breeding system of Atlantic salmon (Salmo salar), with comparisons to other salmonids. Can J Fish Aquat Sci 55:59-76

Fleming I, Agustesson T, Finstad B, Johnsson J, Bjornsson B (2002) Effects of domestication on growth physiology and endocrinology of Atlantic salmon (Salmo salar). Can J Fish Aquat Sci 59:1323-1330

Gall GAE, Huang N (1988) Heritability and selection schemes for rainbow trout body weight. Aquaculture 73:43-56

Gjedrem T (2000) Genetic improvement of cold-water fish species. Aqua Res 31:25-33

Groot C, Margolis L (1991) Pacific salmon life histories. UBC Press, Vancouver

Gross MR (1991) Salmon breeding behavior and life history evolution in changing environments. Ecology 722:11801186

Hails RS, Morley K (2005) Genes invading new populations: a risk assessment perspective. Trends Ecol Evol 20:245-252

Haldane JBS (1957) The cost of natural selection. J Genet 55:511-524

Hamilton W (1967) Extraordinary sex ratios. Science 156: 477-488

Hedrick PW (2001) Invasion of transgenes from salmon or other genetically modified organisms into natural populations. Can J Fish Aquat Sci 58:841-844

Hershberger WK, Myers JM, Iwamoto RN, Macauley WC, Saxton AM (1990) Genetic changes in the growth of coho salmon (Oncorhynchus kisutch) in marine net-pens, produced by ten years of selection. Aquaculture 85:1-4

Howard RD, DeWoody JA, Muir WM (2004) Transgenic male mating advantage provides opportunity for Trojan gene effect in a fish. Proc Nat Acad Sci 101:2934-2938 
Huang YX, Magori K, Lloyd AL, Gould F (2007) Introducing desirable transgenes into insect populations using Ylinked meiotic drive - a theoretical assessment. Evolution 61:717-726

Jaenike J (2001) Sex chromosome meiotic drive. Annu Rev Ecol Syst 32:25-49

Jhingan E, Devlin RH, Iwama GK (2003) Disease resistance, stress response and effects of triploidy in growth hormone transgenic coho salmon. J Fish Biol 63:806823

Kantachuvesiri S, Haley CS, Fleming S, Kurian K, Whitworth CE, Wenham P, Kotelevtsev Y, Mullins JJ (1999) Genetic mapping of modifier loci affecting malignant hypertension in TGRmRen2 rats. Kidney Int 56:414-420

Kapuscinski AR, Hallerman EM (1990) Transgenic fish and public policy: anticipating environmental impacts of transgenic fish. Fisheries 15(2):2-11

Kapuscinski A, Hard J, Paulson K, Neira R, Ponniah A, Kamonrat W, Mwanja W, Fleming I, Gallardo J, Devlin R, Trisak J (2007a) Approaches to assessing gene flow. Chapter 5. In: Kapuscinski AR, Hayes KR, Li S, Dana G (eds) Environmental risk assessment of genetically modified organisms, volume 3: methodologies for transgenic fish. CABI International, Cambridge, pp 151-187

Kapuscinski AR, Hayes KR, Li S, Dana G (2007b) Environmental risk assessment of genetically modified organisms. Vol 3. Methodologies for transgenic fish. CABI International, Oxfordshire

Kitami T, Nadeau JH (2002) Biochemical networking contributes more to genetic buffering in human and mouse metabolic pathways than does gene duplication. Nat Genet 32:191-194

Leggatt RA, Devlin RH, Farrell AP, Randall DJ (2003) Oxygen uptake of growth hormone transgenic coho salmon during starvation and feeding. J Fish Biol 62:1053-1066

Lima SL, Dill LM (1990) Behavioral decisions made under the risk of predation: a review and prospectus. Can $\mathrm{J}$ Zool 68:619-640

Linder C (2001) The influence of genetic background on spontaneous and genetically engineered mouse models of complex diseases. Lab Animal 30:34-39

Maclean N, Laight RJ (2000) Transgenic fish. An evaluation of benefits and risks. Fish Fish 1:146-172

Martinez R, Estrada MP, Berlanga J, Guillen I, Hernandez O, Pimentel R, Morales R, Herrera F, Fuente J et al (1996) Growth enhancement in transgenic tilapia by ectopic expression of tilapia growth hormone. Mol Mar Biol Biotechnol 5:62-70

McClelland EK, Myers JM, Hard JJ, Park LK, Naish KA (2005) Two generations of outbreeding in coho salmon (Oncorhynchus kisutch): Effects on size and growth. Can J Fish Aquat Sci 62:2538-2547

Metz AV, Chynoweth J, Allan AM (2006) Influence of genetic background on alcohol drinking and behavioral phenotypes of 5-HT3 receptor over-expressing mice. Pharmacol Biochem Behav 84:120-127

Muir WM, Howard RD (1999) Possible ecological risks of transgenic organism release when transgenes affect mating success: Sexual selection and the Trojan gene hypothesis. Proc Nat Acad Sci USA 96:13853-13856
Muir WM, Howard RD (2001) Fitness components and ecological risk of transgenic release: a model using Japanese medaka (Oryzias latipes). Am Nat 158:1-16

Muir WM, Howard RD (2002) Assessment of possible ecological risks and hazards of transgenic fish with implications for other sexually reproducing organisms. Transgenic Res 11:101-114

Nadeau JH (2001) Modifier genes in mice and humans. Nature Rev Genet 2:165-174

Nam YK, Cho HJ, Cho YS, Noh JK, Kim CG, Kim DS (2001) Accelerated growth, gigantism and likely sterility in autotransgenic triploid mud loach Misgurnus mizolepis. J World Aquac Soc 32:353-363

Nielsen L, Gurnami M, Catino J, Tyler R (1995) In Wap-Ras transgenic mice, tumour phenotype but not cyclophosphamide-sensitivity is affected by genetic background. Anticancer Res 15:385-392

Ostenfeld TH, McLean E, Devlin RH (1998) Transgenesis changes body and head shape in Pacific salmon. J Fish Biol 52:850-854

Parisi MA, Baldessari AE, Iida MHK, Clarke CM, Doggett B, Shirasawa S, Kapur RP (2003) Genetic background modifies intestinal pseudo-obstruction and the expression of a reporter gene in Hox11L1-/- mice. Gastroenterology 125:1428-1440

Pitkanen TI, Krasnov A, Teerijoki H, Moelsae H (1999) Transfer of growth hormone (GH) transgenes into Arctic charr (Salvelinus alpinus L.) I. Growth response to various GH constructs. Genet Anal Biomol Eng 15:91-98

Rahman MA, Mak R, Ayad H, Smith A, Maclean N (1998) Expression of a novel piscine growth hormone gene results in growth enhancement in transgenic tilapia (Oreochromis niloticus). Transgen Res 7:357-369

Raven PA, Uh M, Sakhrani D, Beckman BR, Cooper K, Pinter J, Leder E, Silverstein J, Devlin RH (2008) Endocrine effects of growth hormone overexpression in transgenic coho salmon. Gen Comp Edocrinol 159:26-37

Reichhardt T (2000) Will souped up salmon sink or swim? Nature 406:10-12

Robertson A, Perea J, Tolmachova T, Thomas PK, Huxley C (2002) Effects of mouse strain, position of integration and tetracycline analogue on the tetracycline conditional system in transgenic mice. Gene 282:65-74

Saunders RL, Fletcher GL, Hew CL (1998) Smolt development in growth hormone transgenic Atlantic salmon. Aquaculture 168:177-194

Siewerdt F, Eisen EJ, Conrad-Brink JS, Murray JD (1998) Gene action of the oMt1a-oGH transgene in two lines of mice with distinct selection backgrounds. J Anim Breed Genet 115:211-226

Siewerdt F, Eisen EJ, Murray JD, Parker IJ (2000) Response to 13 generations of selection for increased 8-week body weight in lines of mice carrying a sheep growth hormonebased transgene. J Anim Sci 78:832-845

Smoker WW, Gharrett AJ, Stekoll MS, Joyce JE (1994) Genetic analysis of size in an anadromous population of pink salmon. Can J Fish Aquat Sci 51:9-15

Stevens ED, Devlin RH (2000) Intestinal morphology in growth hormone transgenic coho salmon. J Fish Biol 56:191-195 
Stokstad E (2002) Engineered fish: friend or foe of the environment? Science 297:1797-1799

Sundström LF, Lohmus M, Johnsson JI, Devlin RH (2004) Growth hormone transgenic salmon pay for growth potential with increased predation mortality. Proc R Soc Lond B Biol Lett 271:350-352

Sundström LF, Lohmus M, Devlin RH (2005) Selection on increased intrinsic growth rates in coho salmon, Oncorhynchus kisutch. Evolution 59:1560-1569

Sundström LF, Lohmus M, Tymchuk WE, Devlin RH (2007) Gene-environment interactions influence ecological consequences of transgenic animals. Proc Nat Acad Sci USA 104:3889-3894

Sundt-Hansen L, Sundstrom LF, Einum S, Hindar K, Fleming IA, Devlin RH (2007) Genetically enhanced growth causes increased mortality in hypoxic environments. Biol Lett 3(2): $165-168$

Suzuki M, Carlson KM, Marchuk DA, Rockman HA (2002) Genetic modifier loci affecting survival and cardiac function in murine dilated cardiomyopathy. Circulation 105:1824-1829

Tiedje JM, Colwell RK, Grossman YL, Hodson RE, Lenski RE, Mack RN, Regal PJ (1989) The planned introduction of genetically engineered organisms: ecological considerations and recommendations. Ecology 70:298-315

Tymchuk WE, Devlin RH (2005) Growth differences among first and second generation hybrids of domesticated and wild rainbow trout (Oncorhynchus mykiss). Aquaculture 245:295-300

Tymchuk WE, Abrahams MV, Devlin RH (2005) Competitive ability and mortality of growth-enhanced transgenic coho salmon fry and parr when foraging for food. Trans Am Fish Soc 134:381-389
Tymchuk WE, Biagi C, Withler RE, Devlin RH (2006) Impact of domestication on growth and behaviour of coho salmon (Oncorhynchus kisutch) and rainbow trout (Oncorhynchus mykiss). Trans Am Fish Soc 135:442-455

Tymchuk WE, Sundström LF, Devlin RH (2007) Growth and survival tradeoffs leading to outbreeding depression in rainbow trout (Oncorhynchus mykiss). Evolution (in press)

Tymchuk WE, Beckman B, Devlin RH (2009) Domestication in fish has genetically altered the expression of hormones involved in the GH / IGF-I growth axis. Endocrinology 150:1809-1816

Valenza-Schaerly P, Pickard B, Walter J, Jung M, Pourcel L, Reik W, Gauguier D, Vergnaud G, Pourcel C (2001) A dominant modifier of transgene methylation is mapped by QTL analysis to mouse chromosome 13. Genome Res 11:382-388

Valosaari K-R, Aikio S, Kaitala V (2008) Male mating strategy and the introgression of a growth hormone transgene. Evol Appl 1:608-619

Venugopal T, Anathy V, Kirankumar S, Pandian TJ (2004) Growth enhancement and food conversion efficiency of transgenic fish Labeo rohita. J Exp Zool 301:477-490

Weichman K, Chaillet JR (1997) Phenotypic variation in a genetically identical population of mice. Mol Cell Biol 17:5269-5274

Weng A, Magnuson T, Storb U (1995) Strain-specific transgene methylation occurs early in mouse development and can be recapitulated in embryonic stem-cells. Development 121:2853-2859

Zhu Z, Li G, He L, Chen S (1985) Novel gene transfer into the fertilized eggs of gold fish (Carassius auratus L. 1758). J Appl Ichthyol 1:31-34 